\title{
A Case- Study of Mucosal Leishmaniasis in the Lip
}

\author{
Jamshid Ayatollahi, ${ }^{1}$ Fatemeh Ayatollahi, ${ }^{2,{ }^{*}}$ Reza Ayatollahi, ${ }^{3}$ and Seyed Hossein Shahcheraghi ${ }^{1,}$ \\ ${ }^{1}$ Infectious Diseases Research Center, Shahid Sadoughi University of Medical Sciences, Yazd, IR Iran \\ ${ }^{2}$ Shahid Sadoughi University of Medical Sciences, Yazd, IR Iran \\ ${ }^{3}$ International Campus, Shahid Sadoughi University of Medical Sciences, Yazd, IR Iran \\ "Corresponding authors: Seyed Hossein Shahcheraghi, Infectious Diseases Research Center, Shahid Sadoughi University of Medical Sciences, Yazd, IR Iran. Tel: +98-3538229200, \\ Fax: +98-3538224100, E-mail: shahcheraghih@gmail.com; Fatemeh Ayatollahi, Shahid Sadoughi University of Medical Sciences, Yazd, IR Iran. Tel: +98-3536255881, Fax: \\ +98-3536250344, E-mail: ayatollahi_mn@yahoo.com
}

Received 2016 May 09; Revised 2016 May 12; Accepted 2016 May 12.

\begin{abstract}
Introduction: Leishmaniasis is a protozoal disease transmitted by sand-fly. The term leishmaniasis encompasses multiple clinical syndromes.

Case Presentation: A 24-year-old male native of Yazd, Iran, was referred from the dental and subsequently to the infectious clinic with complaints of ulcer and swelling over his lower lip for three months. The lesion progressively increased in size over a few weeks and there was no history of fever or other complaints. The lesion had response to treatment with multiple antibiotics by various medical practitioners.

Conclusions: The case was interesting according to the rare location of the lesion since it can be confused with other infectious diseases.
\end{abstract}

Keywords: Leishmaniasis, Lip, Iran, Yazd

\section{Introduction}

Leishmaniasis is a protozoal disease transmitted by sand-fly. The term leishmaniasis encompasses multiple clinical syndromes (1-3). Most notable ones are cutaneous, visceral and mucosal leishmaniasis. Traditionally, the term mucosal leishmaniasis refers to a potentially disfiguring sequel of the New World (America) cutaneous leishmaniasis those results from dissemination of parasites from the skin to the naso-oropharyngeal mucosa $(3,4)$. Leishmaniasis is endemic in Iran (Old World diseases) that usually affects only the skin and mucosal involvement is rare (1, 4-6).

This is to present a case of cutaneous leishmaniasis, which was interesting because of the rare location of the lesion and the fact that it can be confused with other infectious diseases (tuberculosis, leprosy and syphilis), neoplasms, sarcoidosis, and mucosal ulceration from cocaine use.

\section{Case Presentation}

A 24-year-old male referred to the infectious clinic with complaints of swelling and ulcer over his lower lip for three months. The patient had initially noticed a small raised lesion on the right corner of the lower lip which was not itchy and not painful. The swelling progressively increased in size over a few weeks and then its size was constant. The patient had no history of recent travel. There was no similar illness in his family.
There was no history of cigarette smoking or drugs addiction. Also, there was no history of fatigue, weight loss, appetite loss, cough or other complaints. Examination showed a small ulcer, indurated raised lesion on the right corner of the lower lip with well-defined edges but without pain and irritation (Figure 1).

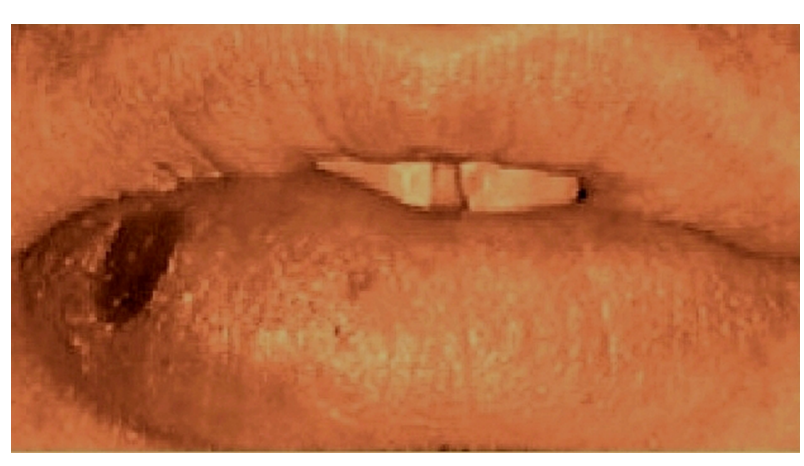

Figure 1. Before Treatment

The vital parameters (temperature and pulse rate) were normal and there was no hepatosplenomegaly or lymphadenopathy. Investigations showed a total leukocyte count of $6900 / \mathrm{mm}^{3}$ with polymorphonuclear $73 \%$ without eosinophilia or thrombocytopenia.

All other hematological and biochemical factors were normal. Erythrocyte sedimentation rate (ESR) and C- 
reactive protein (CRP), were normal. The HIV and VDRL were also tested by ELISA and results were also normal. The Mantoux test was within normal limits ( $5 \mathrm{~mm}$ ).Ultimately, Giemsa-stained thin smear of lesion scraping revealed Leishman bodies (Figure 2) and diagnosis of lip leishmaniasis was made.

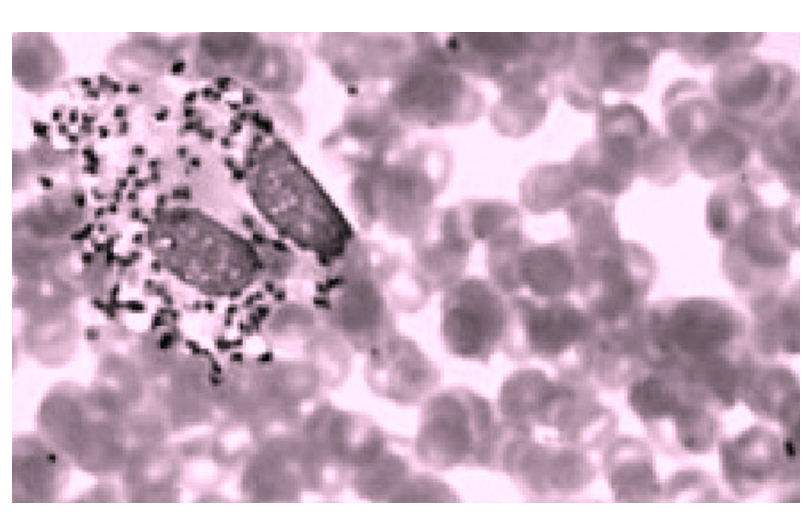

Figure 2. Leishman Bodies

The patient was treated with intramuscularly sodium stibogluconate for 20 days (7). Patient responded very well and two weeks after the start of treatment regression in size started. The lesion completely healed without any residual pigmentation or scarring (Figure 3 ).

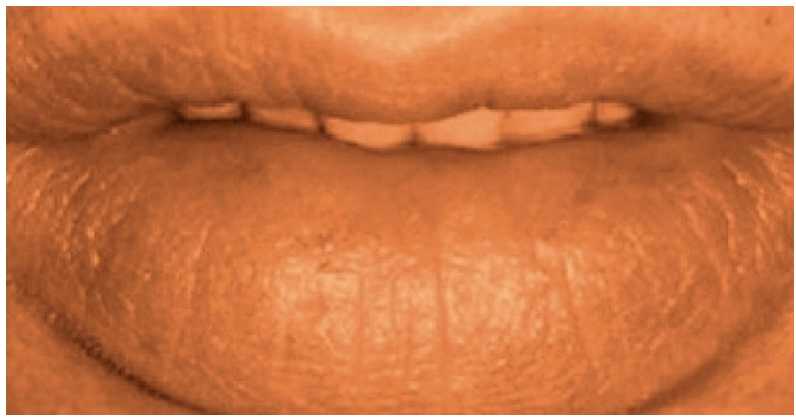

Figure 3. After Treatment

The case was followed up for 12 months and showed no signs of relapse or lesion on other parts of the body.

\section{Discussion}

Lip lesion is seldom mentioned as presentation of cutaneous leishmaniasis in Iran $(5,6)$. Leishmaniasis may have various presentations, and factors affecting the disease spectrum include species and number of parasites, site of bite, and also nutritional status of the patient and the immune status $(5,6)$. Leishmaniasis of the skin may occur anywhere on the body but the usual sites are the exposed parts. Most patients have one or two ulcers, but the lesions may be multiple with various presentations (8). The cases of isolated lip leishmaniasis are seldom reported from Iran (9). The current case is reported since leishmaniasis is often diagnosed presumptively by clinical and epidemiologic criteria.

In endemic regions, considering the increasing spectrum of cutaneous leishmaniasis, a high clinical suspicion should always be observed to make the diagnosis while lesions are in lips $(9,10)$.

This case was interesting because of the rare location of the lesion and the fact that it can be confused with other infectious diseases (tuberculosis, leprosy and syphilis), neoplasms and sarcoidosis.

\section{Acknowledgments}

The authors would like to thank Infectious diseases research center of Yazd Shahid Sadoughi University of Medical Sciences for their kind assistance.

\section{Footnotes}

Authors' Contribution: All authors had equal roles in study design, practical work and writing the manuscript.

Funding/Support: This study was supported by Shahid Sadoughi University of Medical Sciences, Yazd, Iran.

\section{References}

1. Ayatollahi J, Fattahi Bafghi A, Shahcheraghi SH. Rare variants of cutaneous leishmaniasis presenting as eczematous lesions. Med J Islam Repub Iran. 2014;28:71. [PubMed: 25405136].

2. Yaghoobi-Ershadi MR, Hanafi-Bojd AA, Akhavan AA, Zahrai-Ramazani AR, Mohebali M. Epidemiological study in a new focus of cutaneous leishmaniosis due to Leishmania major in Ardestan town, central Iran. Acta Trop. 2001;79(2):115-21. [PubMed: 11369303].

3. Feiz Haddad MH, Ghasemi E, Maraghi S, Tavala M. Identification of Leishmania Species Isolated from Human Cutaneous Leishmaniasis in Mehran, Western Iran Using Nested PCR. Iran J Parasitol. 2016;11(1):65-72. [PubMed: 27095970].

4. Al-Salem WS, Pigott DM, Subramaniam K, Haines LR, Kelly-Hope L, Molyneux DH, et al. Cutaneous Leishmaniasis and Conflict in Syria. Emerg Infect Dis. 2016;22(5):931-3. doi: 10.3201/eid2205.160042. [PubMed: 27088251].

5. Shirian S, Oryan A, Hatam GR, Daneshbod Y. Three Leishmania/L. species-L. infantum, L. major, L. tropica-as causative agents of mucosal leishmaniasis in Iran. Pathog Glob Health. 2013;107(5):267-72. doi: 10.1179/2047773213Y.0000000098. [PubMed: 23916336].

6. Hajjaran H, Mohebali M, Akhavan AA, Taheri A, Barikbin B, Soheila Nassiri S. Unusual presentation of disseminated cutaneous leishmaniasis due to Leishmania major: case reports of four Iranian patients. Asian Pac JTrop Med. 2013;6(4):333-6. doi:10.1016/S1995-7645(13)600668. [PubMed: 23608338]. 
7. Ramos A, Munez E, Garcia-Dominguez J, Martinez-Ruiz R, Chicharro C Banos I, et al. Mucosal leishmaniasis mimicking squamous cell carcinoma in a liver transplant recipient. Transpl Infect Dis. 2015;17(3):488 92. doi: 10.1111/tid.12380. [PubMed: 25816835].

8. Farahmand M, Nahrevanian H, Shirazi HA, Naeimi S, Farzanehnejad Z. An overview of a diagnostic and epidemiologic reappraisal of cutaneous leishmaniasis in Iran. Braz J Infect Dis. 2011;15(1):17-21. [PubMed
21412584]

9. Mohebali M, Malmasi A, Hajjaran H, Jamshidi S, Akhoundi B, Rezaei $M$, et al. Disseminated Leishmaniasis Caused by Leishmania tropica in a Puppy from Karaj, Central Iran. Iran J Parasitol. 2011;6(2):69-73. [PubMed: 22347291].

10. Yaghoobi R, Maraghi S, Bagherani N. Cutaneous leishmaniasis with unusual presentation. Iranian J Parasitol. 2009;4(2):67-9. 